\title{
GSTP1 as a potential predictive factor for adverse events associated with platinum-based antitumor agent-induced peripheral neuropathy
}

\author{
SOU KATAYANAGI, KENJI KATSUMATA, YASUHARU MORI, KATSUNORI NARAHARA, \\ MASATOSHI SHIGOKA, TAKAAKI MATSUDO, MASANORI ENOMOTO, TAKESHI SUDA, \\ TETSUO ISHIZAKI, MASAYUKI HISADA, YUUICHI NAGAKAWA and AKIHIKO TSUCHIDA
}

Department of Digestive and Pediatric Surgery, Tokyo Medical University Hospital, Tokyo 160-0023, Japan

Received May 25, 2018; Accepted October 26, 2018

DOI: $10.3892 / \mathrm{ol} .2019 .9907$

\begin{abstract}
Glutathione S-transferase (GST) exhibits antidotal effects on numerous drugs, including platinum-based antineoplastic drugs. Furthermore, GST Pi 1 (GSTP1) polymorphism is associated with peripheral neuropathy. In the present study, it was determined whether GSTPI can predict adverse events associated with platinum-based antitumor agent-induced peripheral neuropathy among Japanese patients. The subjects included 122 patients, among whom 105 patients had colorectal, 16 had gastric, and one patient had pancreatic cancer. It was indicated that wild type (AA) GSTPI was expressed in 99 patients $(81.1 \%)$, whereas heterozygous (AG) and homozygous (GG) GSTP1 polymorphisms were present in $22(18.0 \%)$ and $1(0.8 \%)$ patients, respectively. Among patients with colorectal cancer, the expression of homozygous GSTPI was observed in 88 patients $(83.8 \%)$, whereas that of heterozygous GSTPI was observed in 17 patients $(16.2 \%)$. Peripheral neuropathy of grade $\geq 3$ occurred in 10 patients (9.5\%) receiving mFOLFOX therapy (a biweekly cycle consisting of a 2 -h infusion of $85 \mathrm{mg} / \mathrm{m}^{2}$ oxaliplatin and $200 \mathrm{mg} / \mathrm{m}^{2}$ leucovorin followed by a bolus administration of $400 \mathrm{mg} / \mathrm{m}^{2} 5$-fluorouracil and a continuous 48 -h infusion of $2,400 \mathrm{mg} / \mathrm{m}^{2} 5$-fluorouracil) for colorectal cancer, which included 6 patients with the AA allele $(6.8 \%)$ and 4 patients with the AG allele (23.5\%). The number of peripheral neuropathy cases of grade $\geq 3$ was increased among patients with the AG allele, compared with patients with the AA allele $(\mathrm{P}=0.032)$. In patients with gastric cancer, the AA and AG types of GSTPl were expressed in $11(68.8 \%)$ and
\end{abstract}

Correspondence to: Dr Sou Katayanagi, Department of Digestive and Pediatric Surgery, Tokyo Medical University Hospital, 7-1 6-chome, Tokyo 160-0023, Japan

E-mail: sou@tokyo-med.ac.jp

Key words: cisplatin, colorectal cancer, gastric cancer, glutathione S-transferase Pi 1, oxaliplatin
$5(31.2 \%)$ patients, respectively. Cisplatin, administered to patients with gastric cancer, did not induce peripheral neuropathy. The aforementioned indicated that GSTPI genetic polymorphism is associated with peripheral neuropathy induced by oxaliplatin treatment for colorectal cancer, and therefore serves as a predictive marker. Furthermore, early dose reduction or drug withdrawal should be implemented depending on the severity of peripheral neuropathy as a potential method for reducing the number of patients discontinuing the drug, due to adverse events involving peripheral neuropathy.

\section{Introduction}

Platinum-containing drugs, platinum-based drugs, vinca alkaloid, taxane-based drugs and bortezomib, a proteasome inhibitor, are cytocidal anticancer agents, which can induce peripheral neuropathy (1). Treatment using the aforementioned drugs must be discontinued when the symptoms of peripheral neuropathy are severe. Furthermore, these adverse events may persist for a long period of time, even following discontinuation of the drug (2). Therefore, management of peripheral neuropathy is important for patients who are treated with the aforementioned drugs.

Glutathione S-transferase (GST) has antidotal effects on numerous drugs, including platinum-based anticancer agents, and genetic polymorphism of GST Pi 1 (GSTP1) has been reported to be associated with the occurrence of peripheral neuropathy (3). GSTP1 is involved in the metabolism of platinum-based anticancer drugs (4), and an association with neurotoxicity has been indicated when large amounts of cisplatin (CDDP) are administered for cancer treatment (5). A study has indicated that FOLFOX therapy using oxaliplatin, which is similar to the platinum anticancer drug CDDP, has a high response rate to colorectal cancer (6). Globally, GSTPI is considered as an indicator of response to chemotherapy and its adverse effects (7), although no definite conclusions have been derived. Reports on the expression of GSTP1 polymorphisms in Japanese patients with gastrointestinal cancer indicated a negligible association with chemotherapy (8-11). In the present study, it was determined whether GSTP1 polymorphism is a 
predictive factor of peripheral neuropathy, which occurs as an adverse effect of exposure to platinum-based anticancer drugs, in Japanese patients with gastric, colorectal, and pancreatic cancer.

\section{Materials and methods}

Patient sample. A total of 122 patients (mean age 65 years; range 35-81 years), whose GSTP1 status was determined at the Tokyo Medical University Hospital (Tokyo, Japan) between April 2005 and December 2008, were included in the present study. Among the following 122 patients: 105 (78 male and 27 female) patients had advanced recurrent colorectal cancer and were receiving mFOLFOX6 therapy (Table I); 16 (12 male and 4 female) patients had advanced recurrent gastric cancer and were receiving chemotherapy, including CDDP (Table II); and 1 female patient had advanced recurrent pancreatic cancer. Chemotherapy for gastric cancer included treatments of 10 patients with S-1/CDDP (SP), 4 patients with CPT-11/CDDP, 1 patient with 5-FU/CDDP (FP) and 1 patient with paclitaxel/CDDP. Treatment with 5-FU/CDDP was used as a chemotherapeutic agent for pancreatic cancer, but this case was firstly treated as pancreatic invasion of stomach cancer; however, the results of the autopsy changed the diagnosis to stomach invasion of pancreatic cancer.

The inclusion criteria were the following: $\geq 18$ years of age, presence of metastatic or non-resectable locally advanced colorectal cancer, gastric and pancreatic cancer, exposure to platinum drugs for chemotherapy and Eastern Cooperative Oncology Group performance status $\leq 2$ (12). The exclusion criteria included the presence of other active cancer types. In patients with colorectal cancer, the expression pattern of GSTPI was examined and the objective tumor response and adverse events that required discontinuation of mFOLFOX6 chemotherapy were identified. In patients with gastric and pancreatic cancer, the expression patterns of GSTP1 and adverse events associated with CDDP chemotherapy were examined. Clinical antitumor effects, according to the Response Evaluation Criteria in Solid Tumors 1.1 guideline (13), and adverse event, according to the National Cancer Institute Common Toxicity Criteria 4.0, were evaluated (14).

The present study was approved by the Ethics Committee of the Tokyo Medical University Hospital (Tokyo, Japan). Furthermore, written informed consent was obtained from the patients prior to the trial. Patients were informed with all the necessary details concerning the study. Direct sequencing was used to analyze GSTP1 polymorphism in 18 healthy individuals and was compared with the results of polymerase chain reaction-restriction fragment length polymorphism (PCR-RFLP), in order to verify consistency of the results obtained using the two methods. The healthy individuals consisted of 14 males and 4 females (median age, 34 years) and they were tested between April and June 2005.

Determination of GSTP1 polymorphism. A single nucleotide substitution $(\mathrm{A} \rightarrow \mathrm{G})$ at position 313 of GSTP1 results in Ile-to-Val substitution at amino acid position 105. Depending on the zygosity [homozygous for the A allele (AA), heterozygous (AG) and homozygous for the $\mathrm{G}$ allele (GG)] of the allele, three common GSTP1 variants, AA/wild type, AG and GG are generated, with the substitution decreasing or abolishing the activity of the encoded enzyme. Genomic DNA was extracted from $200 \mu$ whole blood using a QiaAmp kit (Qiagen Inc., Valencia, CA, USA), according to the manufacturer's protocols. The Ile105Val polymorphism was analyzed using PCR-RFLP as described by Harries et al (15). The $40 \mu \mathrm{l}$ reaction mixture contained $5 \mu \mathrm{l}$ cell lysate, which was used as a template, $200 \mathrm{ng}$ of each primer, 105 forward, 5'-ACCCCAGGCTCTATGGGAA-3' and 105 reverse, 5'-TGAGGGCACAAGAAGCCCCT-3'. The primerse were made and supplied by Eurofinsgenomics (Ohta, Tokyo, Japan), $2.0 \mathrm{mM}$ magnesium chloride and 1.5 U Taq DNA polymerase (both from Applied Biosystems; Thermo Fisher Scientific, Inc., Waltham, MA, USA). Initial denaturation was performed at $95^{\circ} \mathrm{C}$ for $5 \mathrm{~min}$. The thermocycling conditions (30 cycles) were: Primer annealing at $55^{\circ} \mathrm{C}$ for $30 \mathrm{sec}$, polymerization at $72^{\circ} \mathrm{C}$ for $30 \mathrm{sec}$, and strand separation at $94^{\circ} \mathrm{C}$ for $30 \mathrm{sec}$. A final polymerization step at $72^{\circ} \mathrm{C}$ for 5 min was included to complete elongation. At the annealing temperature, the sample was digested using $5 \mathrm{U} / \mathrm{ml} B s m \mathrm{AI}$ (New England Biolabs, Inc., Ipswich, MA, USA), and the fragments were separated on a 3.0\% Metaphor agarose gel (FMC BioProducts, Philadelphia, PA, USA) and visualized following staining with ethidium bromide at $55^{\circ} \mathrm{C}$ for $12 \mathrm{~h}$.

Direct sequencing of PCR products. PCR products $(\sim 50 \mu \mathrm{l})$ were purified using a QIAquick PCR purification kit (Qiagen $\mathrm{GmbH}$, Hilden, Germany), according to the manufacturer's protocols, prior to sequencing. The concentration of the PCR product was estimated on a $2 \%$ agarose gel. The product $(\sim 250 \mathrm{ng})$ was used as the template in a double-stranded (ds) cycle sequencing reaction using the ds-DNA cycle sequencing system (Gibco; Thermo Fisher Scientific, Inc.), according to the manufacturer's protocols, and was labeled with $(\gamma 32 \mathrm{P})$ dATP. Sequencing was performed from both directions separately following PCR with the 105 forward and 105 reverse primers according to the manufacturer's protocols. Cycling conditions included initial denaturation at $94^{\circ} \mathrm{C}$ for 5 min followed by 20 cycles of denaturation at $94^{\circ} \mathrm{C}$ for $30 \mathrm{sec}$, primer annealing at $47^{\circ} \mathrm{C}$ for $60 \mathrm{sec}$ and polymerization at $72^{\circ} \mathrm{C}$ for $60 \mathrm{sec}$. The reaction was completed by 10 cycles of denaturation at $94^{\circ} \mathrm{C}$ for $30 \mathrm{sec}$ and polymerization at $72^{\circ} \mathrm{C}$ for $60 \mathrm{sec}$. The PCR product sequence was entrusted to commercial-based vendors (Eurofinsgenomics).

Statistical analysis. The data are presented as the mean values. The SPSS 24.0 software (IBM Corp., Armonk, NY, USA) was used for statistical analysis. The $\chi^{2}$ test was performed for comparing response rates between the groups and an unpaired Student's t-test for comparing the means. $\mathrm{P}<0.05$ was considered to indicate a statistically significant difference.

\section{Results}

Analyzing GSTP1 polymorphism in healthy individuals. Analysis of GSTPI polymorphism of 18 healthy individuals using direct sequencing revealed that 15 patients harbored the AA allele, 2 harbored the AG allele and 1 harbored the GG allele (Fig. 1). All GSTP1 single nucleotide polymorphism 
Table I. Clinical information of patients with colorectal cancer.

\begin{tabular}{|c|c|c|c|}
\hline \multirow[b]{2}{*}{ Factors } & \multicolumn{2}{|c|}{ Genotype } & \multirow[b]{2}{*}{ P-value } \\
\hline & AA $(n=88)$ & $\mathrm{AG}(\mathrm{n}=17)$ & \\
\hline Sex & & & $0.04^{\mathrm{a}}$ \\
\hline Male & 62 & 16 & \\
\hline Female & 26 & 1 & \\
\hline Median age, years & 66 & 60 & 0.08 \\
\hline Primary site & & & 0.27 \\
\hline Colon & 49 & 7 & \\
\hline Rectum & 39 & 10 & \\
\hline Stage $^{b}$ & & & 0.63 \\
\hline II & 10 & 1 & \\
\hline III & 28 & 7 & \\
\hline IV & 50 & 9 & \\
\hline $\begin{array}{l}\text { Number of cycles of } \\
\text { CDDP treatment }\end{array}$ & & & 0.05 \\
\hline Adj & 3 & 1 & \\
\hline $1 \mathrm{st}$ & 49 & 3 & \\
\hline 2 nd & 26 & 11 & \\
\hline Following 3rd & 10 & 2 & \\
\hline Cycle (median) & 10 & 10 & 0.81 \\
\hline Timeframe & & & 0.63 \\
\hline Synchronous & 47 & 8 & \\
\hline Metachronous & 41 & 9 & \\
\hline Cancellation reason & & & 0.61 \\
\hline PD & 35 & 4 & \\
\hline Toxicity with PN & 25 & 7 & \\
\hline Toxicity without PN & 18 & 4 & \\
\hline Other & 10 & 2 & \\
\hline
\end{tabular}

${ }^{\mathrm{a}} \mathrm{P}<0.05$. ${ }^{\mathrm{b}}$ Staging according to the International Union Against Cancer Tumor-Node-Metastasis classification 8th edition (39). Adj, adjuvant chemotherapy; PD, progressive disease; PN, peripheral neuropathy.

(SNP) sites contained the ATC sequence for the homozygous AA allele, and the A/GTC (Fig. 2A) and GTC (Fig. 2B) sequences at the SNP position. These results of the direct sequencing were in accordance with those obtained using PCR-RFLP, thereby demonstrating the accuracy of the GST analysis method.

Expression of AA type and polymorphic GSTP1. In the entire study group, GSTPI harboring the AA allele (Ile/Ile) was expressed in 99 patients $(81.2 \%)$. Furthermore, the $\mathrm{AG}$ (Ile/Val) and GG (Val/Val) variants of GSTP1 were expressed in $22(18.0 \%)$ and $1(0.8 \%)$ patients, respectively. The mean age of the patients with AA and AG in GSTP1 were 65.3 and 61.1 years, respectively, indicating no significant difference $(\mathrm{P}>0.05)$ between the AA and AG group. In patients with colorectal cancer, the AA (Ile/Ile) and AG (Ile/Val) polymorphic GSTP1 were expressed in $88(83.8 \%)$ and $17(16.2 \%)$ cases, respectively. In patients with gastric
Table II. Clinical information of patients with gastric cancer.

\begin{tabular}{lccc}
\hline & \multicolumn{2}{c}{ Genotype } & \\
\cline { 2 - 3 } Factors & AA $(\mathrm{n}=11)$ & AG (n=5) & P-value \\
\hline Sex & 9 & 3 & 0.37 \\
Male & 2 & 2 & \\
Female & 69 & 62 & 0.17 \\
Median age, years & & & 0.71 \\
Number of cycles of & & & \\
CDDP treatment & 7 & 4 & \\
1st & 1 & 0 & \\
2nd & 3 & 1 & \\
Following 3rd & 2 & 2 & 0.77 \\
Median cycle & & & 0.48 \\
Cancellation reason & 7 & 4 & \\
PD & 0 & 0 & \\
Toxicity with PN & 4 & 1 & \\
Toxicity without PN & 0 & 0 & \\
Other & & & \\
\hline
\end{tabular}

$\mathrm{PD}$, progressive disease; PN, peripheral neuropathy.

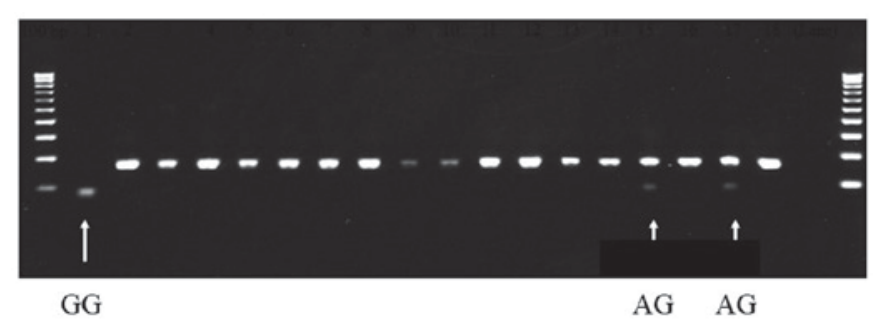

Figure 1. Genotyping of GSTP1 polymorphism in healthy volunteers Polymerase chain reaction-restriction fragment length polymorphism performed on samples collected from 18 healthy volunteers indicated that the homozygous GSTP1 variant was present in one (arrow in lane 1) and heterozygous GSTP1 polymorphism in two patients (arrows in lanes 15 and 17). GSTP1, glutathione S-transferase Pi 1.
A

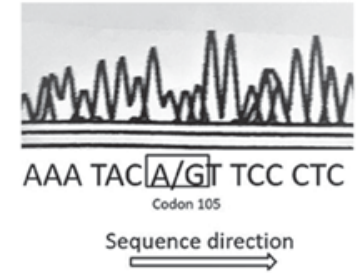

B

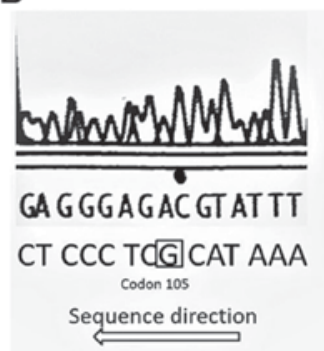

Figure 2. All GSTP1 SNP sites contained the ATC sequence for the homozygous AA allele, and the (A) A/GTC and (B) GTC sequences at the SNP position. SNP, single nucleotide polymorphism.

cancer, the AA group and heterozygous polymorphic GSTP1 were expressed in $11(68.8 \%)$ and $5(31.2 \%)$ cases, respectively. 
Patients with colorectal cancer. The proportion of female patients with colorectal cancer carrying the AA genotype of GSTP1 was significantly higher than those with the AG form ( $\mathrm{P}=0.04$; Table I). There was no significant difference in the number of doses of mFOLFOX6 administered between the two groups, 9.6 times for the wild type GSTP1 and 10.3 times for AG-GSTP1 ( $\mathrm{P}=0.81$; Table I). Chemotherapy was discontinued due to disease progression in 39 patients $(37.1 \%)$, peripheral neuropathy in 32 patients (30.5\%), adverse events other than peripheral neuropathy in 22 patients $(21.0 \%)$ and for other reasons in 12 patients (11.4\%). Blood toxicity of grade $\geq 3$ occurred in 13 cases harboring the AA genotype (14.8\%) and 3 cases harboring the AG polymorphism (17.6\%); however, the difference was not significant $(\mathrm{P}=0.76$; Table III). Peripheral neuropathy of grade $\geq 3$ was observed in 10 patients $(9.5 \%)$, of which 6 patients harbored the AA $(6.8 \%)$ and 4 harbored the AG genotype of GSTPI (23.5\%). Peripheral neuropathy of grade $\geq 3$ was observed at significantly increased rates in patients with AG polymorphism, compared with patients with the AA genotype $(\mathrm{P}=0.032$; Table IV). The two groups did not indicate a significant difference in terms of non-hematological toxicity other than peripheral neuropathy of grade $\geq 3$, with 12 patients of the AA type (13.6\%) and 3 of the AG type $(17.6 \%)$ indicating this effect $(\mathrm{P}=0.71$; Table $\mathrm{V})$. The therapeutic effect of mFOLFOX6 (complete response/partial response/stable disease/progressive disease/cannot be evaluated) was $7 / 23 / 34 / 21 / 3$, respectively, for the AA type and $0 / 3 / 6 / 5 / 3$, respectively, for the AG type patients. The aforementioned results were not statistically significant $(\mathrm{P}=0.67$; Table VI).

Patients with gastric cancer. In patients with gastric cancer, GSTP1 with the AA genotype was expressed in 11 patients (68.8\%), whereas the AG polymorphic version was expressed in 5 patients $(31.2 \%)$ (Table II). No significant differences were observed in the clinicoepidemiological data between the two groups. The median number of CDDP treatments was 2 cycles (range, 1-8 cycles). There was no significant difference between AA and AG genotype of GSTP1 in terms of hematological toxicity during chemotherapy with CDDP, with 4 patients with AA type $(36.4 \%)$ and no patient with AG type exhibiting hematological toxicity events of grade $\geq 3$ ( $P=0.12$; Table VII). Furthermore, non-hematological toxicity of grade $\geq 3$ occurred in 4 cases harboring the AA allele $(36.3 \%)$ and 2 cases bearing the AG allele $(40.0 \%)$, exhibiting no significant difference between the two groups ( $\mathrm{P}=0.89$; Table VIII). In the present study, no patient exhibited peripheral neuropathy.

Patients with pancreatic cancer. With respect to adverse events of FP therapy, the GG version of GSTP1 was observed only in a 71-year-old female patient with stage IV pancreatic cancer. They had grade 3 myelosuppression and gastrointestinal symptoms without peripheral neuropathy. Following chemotherapy, they developed progressive disease. The ratios of the various forms of GSTP1 detected in patients with pancreatic cancer were as follows: Wild type, $44.7 \%$; heterozygous polymorphism, $41.7 \%$; and homozygous polymorphism, $13.6 \%$ (16).
Table III. GSTP1 genotypes and hematological toxicity of grade $\geq 3$ in patients with colorectal cancer.

\begin{tabular}{lrrr}
\hline & \multicolumn{2}{c}{$\begin{array}{c}\text { Hematological } \\
\text { toxicity }\end{array}$} & \\
\cline { 2 - 3 } GSTP1 genotype & Yes & No & P-value \\
\cline { 2 - 3 } & & & 0.76 \\
AA & 13 & 75 & \\
AG & 3 & 14 & \\
\hline
\end{tabular}

GSTP1, glutathione S-transferase Pi 1. Grade, National Cancer Institute Common Toxicity Criteria 4.0.

Table IV. GSTP1 genotypes and peripheral neuropathy in patients of grade $\geq 3$ with colorectal cancer.

\begin{tabular}{llll}
\hline & \multicolumn{2}{l}{ Peripheral neuropathy } & \\
\cline { 2 - 3 } GSTP1 genotype & Yes & No & P-value \\
\hline & & & 0.032 \\
AA & 6 & 82 & \\
AG & 4 & 13 & \\
\hline
\end{tabular}

GSTP1, glutathione S-transferase Pi 1. Grade, National Cancer Institute Common Toxicity Criteria 4.0.

Table V. GSTP1 genotypes and adverse events in grade $\geq 3$ patients with colorectal cancer.

\begin{tabular}{lrcr}
\hline & \multicolumn{2}{c}{$\begin{array}{l}\text { Non-hematological toxicity } \\
\text { except peripheral neuropathy }\end{array}$} & \\
\cline { 2 - 3 } GSTP1 genotype & Yes & No & P-value \\
\cline { 2 - 3 } & & & 0.71 \\
AA & 12 & 76 & \\
AG & 3 & 14 & \\
\hline
\end{tabular}

GSTP1, glutathione S-transferase Pi 1. Grade, National Cancer Institute Common Toxicity Criteria 4.0

\section{Discussion}

A total of two SNPs in GSTPl have been reported $(17,18)$, one of which is the $A \rightarrow G$ substitution at nucleotide position 313 observed in the present study, where the amino acid Ile is replaced by Val at codon 105 . The other is the $\mathrm{C} \rightarrow \mathrm{T}$ replacement at the nucleotide 341, in which the amino acid Ala at codon 114 is replaced by Val. These SNPs are associated with the sensitivity and adverse events accompanying oxaliplatin treatment (4). In the present study, the SNP status of GSTP1 was determined based on the report of Lecomte et al (19), who indicated that oxaliplatin-induced neurotoxicity occurs 
Table VI. Genotypes and the therapeutic effect of mFOLFOX6.

\begin{tabular}{lccc}
\hline & \multicolumn{2}{c}{ Genotype } & \\
\cline { 2 - 3 } Patient response & AA (n=88) & AG (n=17) & P-value \\
\hline & 7 & 0 & 0.67 \\
CR & 23 & 3 & \\
PR & 34 & 6 & \\
SD & 21 & 5 & \\
PD & 3 & 3 & \\
NE & & & \\
\hline
\end{tabular}

CR, complete response; $\mathrm{PR}$, partial response; $\mathrm{SD}$, stable disease; $\mathrm{PD}$, progressive disease; NE, cannot be evaluated.

Table VII. GSTP1 genotypes and hematological toxicity of grade $\geq 3$ in patients with gastric cancer.

\begin{tabular}{lccc}
\hline & \multicolumn{2}{l}{ Hematological toxicity } & \\
\cline { 2 - 3 } GSTP1 genotype & Yes & No & P-value \\
\hline & & & 0.12 \\
AA & 4 & 7 & \\
AG & 0 & 5 & \\
\hline
\end{tabular}

GSTP1, glutathione S-transferase Pi 1. Grade, National Cancer Institute Common Toxicity Criteria 4.0

Table VIII. GSTP1 genotypes and non-hematological toxicity of grade $\geq 3$ in patients with gastric cancer.

\begin{tabular}{lccc}
\hline & \multicolumn{2}{c}{$\begin{array}{l}\text { Non-hematological toxicity } \\
\text { except peripheral neuropathy }\end{array}$} & \\
\cline { 2 - 3 } GSTP1 genotype & Yes & No & P-value \\
\hline & & & 0.89 \\
AA & 4 & 7 & \\
AG & 2 & 3 & \\
\hline
\end{tabular}

GSTP1, glutathione S-transferase Pi 1 .

through the variant codon 105 of GSTPI. To the best of our knowledge, this is the first report in which GSTP1 polymorphism was determined using PCR-RFLP for patients with colorectal, gastric or pancreatic cancer during the same timeframe.

Currently, platinum-based agents are being used as key drugs in chemotherapy for colon and gastric cancer. Oxaliplatin in mFOLFOX therapy is commonly used for non-resectable and advanced colon cancer (20). Similarly, CDDP and oxaliplatin are frequently used as important chemotherapeutic drugs for gastric cancer (21). However, it was repeatedly observed that one of the adverse effects of using platinum-based agents was the development of peripheral neuropathy, which significantly determined the course of subsequent treatment.

GST is a detoxification enzyme that eliminates drugs and toxins by binding to them in vivo (4). Any abnormality in GST affects platinum detoxification, which is presumed to result in an increased frequency of peripheral neuropathy (19). Administration of large doses of glutathione also reduces the frequency of neuropathy (5). Furthermore, GST- $\pi$, a sub-class of GST, is associated with the sensitivity of platinum-based agents (4). Moscow et al (22) reported that in all cases of colorectal cancer, GST- $\pi$ expression was increased by 3.7-fold in the tumor tissue, compared with its matched control tissue. DNA-damaging agents crosslink the DNA in the cell, and intracellular glutathione eliminates DNA-damaging agents via ATP-binding cassette transporters. Therefore, GST- $\pi$ serves an important role in mediating the interaction between the agent and glutathione (23). Additionally, glutathione administration suppresses neurotoxicity and blocks apoptotic cell death induced by tumor protein p53-dependent activation (24).

AA (Ile/Ile), AG (Ile/Val) and GG (Val/Val) types of GSTP1 were observed in 58, 35, and 7\% of Europeans, respectively in 2007 (7). According to the North American Gastrointestinal Intergroup Trial N9741 in USA, AA, AG and GG included 194 (41.9\%), 220 (47.5\%) and 49 (10.6\%) patients out of the 463 patients included in the trial (25).

The prevalence of AA, AG and GG genotypes was 75.3, 22.9 and $1.8 \%$, respectively, in Chinese populations (26). It was lower ratio in AG and GG than Europeans and American. The present study also identified an increased proportion of AA type patients (81.1\%), compared with AG (18\%) and GG $(0.8 \%)$ type patients in Japan. It may be a racial difference. Genetic polymorphism of GSTP1 is a predictive factor of oxaliplatin-induced peripheral neuropathy in patients with colorectal cancer (27). Furthermore, patients with GG polymorphism of GSTP1 were more likely to discontinue FOLFOX due to neurotoxicity ( 24 vs. $10 \%$; $P=0.01$ ) (7). AG or GG type genetic polymorphism has been reported to develop stronger disorder (Grade 3 and 4) for neuropathy (25). The results of the present study also revealed a significantly increased onset of peripheral neuropathy of grade 3 or 4 in AG type patients, compared with AA type patients $(\mathrm{P}=0.032)$, indicating that GSTP1 may serve as a potential marker of adverse events. If GSTP1 status could be used to determine patients with an increased likelihood of peripheral neuropathy onset, it would be easier for physicians and pharmacists to provide accurate instructions and check for subjective symptoms. To prevent adverse effects, a 'stop and go' method involving withdrawal of oxaliplatin alone (28) may be effective. Furthermore, this would help pre-determine the number of cycles of FOLFOXIRI-Bev, for which a maximum of 12 cycles was currently used in the Triplet plus Bevacizumab trial (29). FOLFIRI treatment, without oxaliplatin, as the first-line therapy may be therapeutically beneficial. Evaluating the risk of peripheral neuropathy based on the background of the patient and deciding on an individualized treatment strategy are also important. However, peripheral neuropathy of grade $\geq 3$ has been reported to be more common for individuals with AA (Ile/Ile) type, compared with AG (Ile/Val) and GG (Val/Val) types (19), however these results are controversial. 
These conflicting results may be attributed to the involvement of external factors other than GSTP1, which may include the following: XRCC1 genetic polymorphism (30), exacerbation of symptoms due to hand-foot syndrome and weakened antidotal effects of oxaliplatin owing to hepatic failure. Therefore, this aspect warrants further investigation.

A number of studies indicate that GSTP1 is a prognostic factor $(4,26,31)$. The AG and GG types of GSTP1 have a high response rate to FOLFOX treatment, and longer progression-free (12.0 vs. 6.0 months, $\mathrm{P}<0.01)$ and overall (25.0 vs. 16.0 months, $\mathrm{P}<0.01$ ) survivals were observed in $A G$ and GG types (26); however, other studies have reported that the prognosis of AA homozygotes of GSTP1-105 (Ile/Ile) is poor $(4,26,31)$. Furthermore, according to a previous study, there has been no significant association reported between the expression of GSTP1 and the therapeutic effect, as the group with GSTP1 overexpression was resistant to platinum-based drugs with poor prognosis (32). This indicates that GSTPI may be beneficial in designing treatment strategies.

In the present study, there were no significant differences in hematological or non-hematological toxicity during chemotherapy with CDDP for gastric cancer, with respect to GSTPI expression. Furthermore, there were no adverse events of peripheral neuropathy. Peripheral neuropathy associated with CDDP treatment has been reported to occur in a dose-dependent manner, with neurotoxic events starting to appear at a total dose of $250-500 \mathrm{mg} / \mathrm{m}^{2}$ (body surface) (33). Additionally, these events occur in $50 \%$ of patients at a total dose of $900 \mathrm{mg} / \mathrm{m}^{2}$ and in $100 \%$ of patients at a total dose of $1,300 \mathrm{mg} / \mathrm{m}^{2}(33)$. A single dose of CDDP for gastric cancer in SP therapy is $60 \mathrm{mg} / \mathrm{m}^{2}$, and therefore, the total dose was not notably high, which may have been one of the reasons that patients did not develop peripheral neuropathy in the present study. Liu et al (34) reported that in gastric cancer patients treated with oxaliplatin, they harboring AG and GG polymorphisms of GSTP1 had stronger neurological, gastrointestinal disorders and hematologic toxicity (Grade $\geq 3$ ) than those of AA.

However, this remains controversial with a number of reports stating that GSTP1 is not a predictive factor of the efficacy of chemotherapy (35). A number of reports also indicated that GSTP1 is not a prognostic factor $(8,36,37)$. In addition to the reports on the association of GSTPI with the metabolism of anticancer agents, a number of previous studies reported that the GG polymorphism is significantly more common in patients with gastric cancer, compared with healthy individuals, indicating that GSTP1 is associated with gastric carcinogenesis $(9,10)$. In particular, the aforementioned association was prominent among Asians (11). Furthermore, GSTPI has been associated with the onset of gastric cancer (38); however, it was not associated with disease prognosis $(35,36)$. Owing to the increase in the number of novel anticancer drugs and advancements in the methods of administration, cancer prognosis has improved significantly (21). Therefore, GSTP1 status alone may not be a viable prognostic factor.

The limitation of the present clinical study was that the platinum-based drug was not administered at the same time for patients with colon and gastric cancer as the first-line of treatment. Therefore, it was not possible to achieve a good comparison of progression-free or overall survival between the two groups.
The results of the present study indicate that the GSTPI genetic polymorphism is associated with peripheral neuropathy induced by oxaliplatin administered for treating colon cancer, and, therefore may be an effective prognostic marker. Early dose reduction or cessation according to the severity of peripheral neuropathy may reduce the number of patients who discontinued treatment, due to peripheral neuropathy. However, the frequency of peripheral neuropathy in patients harboring the AG polymorphism observed in the present study was low (22\%), compared with patients from Western countries $(51 \%)$ reported in an earlier study (38). Therefore, it is unclear whether clinically sufficient results were obtained. To the best of our knowledge, the number of conclusive reports on factors that predict adverse effects of platinum-based agents for gastric cancer is limited; therefore future investigation on the aforementioned topic is required.

\section{Acknowledgements}

The authors would like to thank Dr. Kazuhiko Kasuya (Department of Digestive and Pediatric Surgery, Tokyo Medical University Hospital) for the support and helpful suggestions for the present study.

\section{Funding}

The present study was funded by a grant from Tokyo Medical University (Tokyo, Japan).

\section{Availability of data and material}

The datasets acquired during and/or analyzed during the current study are available from the corresponding author on reasonable request.

\section{Authors' contributions}

SK designed the study and wrote the initial draft of the manuscript. KK contributed to analysis and interpretation of data and assisted in the preparation of the manuscript. All other authors (YM, KN, MS, TM, ME, TS, TI, MH, YN and AT) contributed to data collection and interpretation, and critically reviewed the manuscript. All authors have approved the final version of the manuscript and have agreed to be accountable for all aspects of the study, including answering of questions related to the accuracy or integrity of the data.

\section{Ethics approval and consent to participate}

This study was approved by the Ethics Committee of Tokyo Medical University hospital and written informed consent was obtained from all patients.

\section{Patient's consent for publication}

Consent for publication was obtained from all participants.

\section{Competing interests}

The authors declare that they have no competing interests. 


\section{References}

1. Mitsuma A and Ando Y: Genetic backgrounds of peripheral neuropathy induced by oxaliplatin. Nihon Yakurigaku Zasshi 141: 62-65, 2013 (In Japanese).

2. Yamasaki K, Nagase M, Tamagawa $H$, Ueda $S$, Tamura $T$, Murata K,Eguchi Nakajima T,Baba E, Tsuda M, Moriwaki T, et al: Randomized phase III study of bevacizumab plus FOLFIRI and bevacizumab plus mFOLFOX6 as first-line treatment for patients with metastatic colorectal cancer (WJOG4407G). Ann Oncol 27: 1539-1546, 2016.

3. Lecomte T, Landi B, Beaune P, Laurent-Puig P and Loriot MA: Glutathione S-transferase P1 polymorphism (Ile105Val) predicts cumulative neuropathy in patients receiving oxaliplatin-based chemotherapy. Clin Cancer Res 12: 3050-3056, 2006.

4. Stoehlmacher J, Park DJ, Zhang W, Groshen S, Tsao-Wei DD, $\mathrm{Yu}$ MC and Lenz HJ: Association between glutathione S-transferase P1, T1 and M1 genetic polymorphism and survival of patients with metastatic colorectal cancer. J Natl Cancer Inst 94: 936-942, 2002.

5. Sumiyoshi $Y$, Hashine $K$, Kasahara $K$ and Karashima $T$ : Glutathione chemoprotection therapy against CDDP-induced neurotoxicity in patients with invasive bladder cancer. Gan To Kagaku Ryoho 23: 1506-1508, 1996 (In Japanese).

6. De Gramont A, Vignoud J, Tournigand C, Louvet C, André T, Varette C, Raymond E, Moreau S, Le Bail N and Krulik M: Oxaliplatin with high-dose leucovorin and 5-fluorouracil 48-hour continuous infusion in pretreated metastatic colorectal cancer. Eur J Cancer 33: 214-219, 1997.

7. Le Morvan V, Smith D, Laurand A, Brouste V, Bellott R, Soubeyran I, Mathoulin-Pelissier S and Robert J: Determination of ERCC2 Lys751Gln and GSTP1 Ile105Val gene polymorphisms in colorectal cancer patients: Relationships with treatment outcome. Pharmacogenomics 8: 1693-1703, 2007.

8. Meulendijks D, Rozeman EA, Cats A, Shkorska K, Joerger M, Deenen MJ, Beijnen JH and Schellens JHM: Pharmacogenetic variants associated with outcome in patients with advanced gastric cancer treated with fluoropyrimidine and platinum-based triplet combinations: A pooled analysis of three prospective studies. Pharmacogenomics J 17: 441-451, 2017.

9. de Araújo RM, de Melo CF, Neto FM, da Silva JN, Soares LF, de Arruda Cardoso Smith M, Sousa EC Jr, Burbano RM, de Medeiros AC and Lima EM: Association study of SNPs of genes IFNGR1 (rs137854905), GSTT1 (rs71748309), and GSTP1 (rs1695) in gastric cancer development in samples of patient in the northern and northeastern Brazil. Tumour Biol 35: 4983-4986, 2014.

10. Chen ZH, Xian JF and Luo LP: Association between GSTM1, GSTT1, and GSTP1 polymorphisms and gastric cancer risk, and their interactions with environmental factors. Genet Mol Res 16, 2017.

11. Ma Y, Wei X, Han G, Xue M, Li G and Li Y: Glutathione S-transferase P1 Ile105Val polymorphism contributes to increased risk of gastric cancer in East Asians. Tumour Biol 34: 1737-1742, 2013

12. Cancer Therapy Evaluation Program: Common Toxicity Criteria (CTC) Version 2.0. https://ctep.cancer.gov/protocolDevelopment/electronic_applications/docs/ctcv2nom-4-30-99-final3.pdf. Accessed April 30, 1999.

13. Eisenhauer EA, Therasse P, Bogaerts J, Schwartz LH, Sargent D, Ford R, Dancey J, Arbuck S, Gwyther S, Mooney M, et al: New response evaluation criteria in solid tumours: Revised RECIST guideline (version 1.1). Eur J Cancer 45: 228-247, 2009.

14. U.S. Department of Health and Human Services, National Institutes of Health, National Cancer Institute: Common terminology criteria for adverse events (CTCAE) Version 4.0. https:// evs.nci.nih.gov/ftpl/CTCAE/CTCAE_4.03/CTCAE_4.03_201006-14_QuickReference_5x7.pdf. Accessed May 28, 2009.

15. Harries LW, Stubbins MJ, Forman D, Howard GC and Wolf CR: Identification of genetic polymorphisms at the glutathione S-transferase Pi locus and association with susceptibility to bladder, testicular and prostate cancer. Carcinogenesis 18: 641-644, 1997.

16. Jiao L, Bondy ML, Hassan MM, Chang DZ, Abbruzzese JL, Evans DB, Smolensky MH and Li D: Glutathione S-transferase gene polymorphisms and risk and survival of pancreatic cancer. Cancer 109: 840-848, 2007.

17. Cowell IG, Dixon KH, Pemble SE, Ketterer B and Taylor JB: The structure of the human glutathione S-transferase pi gene. Biochem J 255: 79-83, 1988.
18. Kano T, Sakai M and Muramatsu M: Structure and expression of a human class pi glutathione S-transferase messenger RNA. Cancer Res 47: 5626-5630, 1987.

19. Lecomte T, Landi B, Beaune P, Laurent-Puig P and Loriot M: Glutathione S-transferase P1 polymorphism (Ile105Val) predicts cumulative neuropathy in patients receiving oxaliplatin-based chemotherapy. Clin Cancer Res 12: 3050-3056, 2006.

20. Cheeseman SL, Joel SP, Chester JD, Wilson G, Dent JT, Richards FJ and Seymour MT: A 'modified de Gramont' regimen of fluorouracil, alone and with oxaliplatin, for advanced colorectal cancer. Br J Cancer 87: 393-399, 2002.

21. Koizumi W, Narahara H, Hara T, Takagane A, Akiya T, Takagi M, Miyashita K, Nishizaki T, Kobayashi O, Takiyama W, et al: S-1 plus cisplatin versus S-1 alone for first-line treatment of advanced gastric cancer (SPIRITS trial): A phase III trial. Lancet Oncol 9: 215-221, 2008.

22. Moscow JA, Fairchild CR, Madden MJ, Ransom DT, Wieand HS, O'Brien EE, Poplack DG, Cossman J, Myers CE and Cowan KH: Expression of anionic glutathione-S-transferase and P-glycoprotein genes in human tissues and tumors. Cancer Res 49: 1422-1428, 1989.

23. Oki E, Kakeji Y, Ohgaki K, Saeki K, Morita M, Emi Y and Maehara Y: Impact of single nucleotide polymorphisms in glutathione S transferase gene GSTP1 in the treatment with oxaliplatin based chemotherapy. Gan To Kagaku Ryoho 35: 1094-1096, 2008 (In Japanese).

24. Park SA, Choi KS, Bang JH, Huh K and Kim SU: Cisplatin-induced apoptotic cell death in mouse hybrid neurons is blocked by antioxidants through suppression of cisplatin-mediated accumulation of p53 but not of Fas/Fas ligand. J Neurochem 75: 946-953, 2000.

25. McLeod HL, Sargent DJ, Marsh S, Green EM, King CR, Fuchs CS, Ramanathan RK, Williamson SK, Findlay BP, Thibodeau SN, et al: Pharmacogenetic predictors of adverse events and response to chemotherapy in metastatic colorectal cancer: Results from North American gastrointestinal intergroup Trial N9741. J Clin Oncol 28: 3227-3233, 2010.

26. Chen YC, Tzeng CH, Chen PM, Lin JK, Lin TC, Chen WS Liang JK, Wang HS and Wang WS: Influence of GSTP1 1105V polymorphism on cumulative neuropathy and outcome of FOLFOX-4 treatment in Asian patients with colorectal carcinoma. Cancer Sci 101: 530-535, 2010.

27. Inada M, Sato M, Morita S, Kitagawa K, Kawada K, Mitsuma A, Sawaki M, Fujita K and Ando Y: Association between oxaliplatin-induced peripheral neuropathy and polymorphisms of the ERCC1 and GSTP1 genes. Int J Clin Pharmacol Ther 48: 729-734, 2010

28. Tournigand C, Cervantes A, Figer A, Lledo G, Flesch M, Buyse M, Mineur L, Carola E, Etienne PL, Rivera F, et al: OPTIMOX1: A randomized study of FOLFOX4 or FOLFOX7 with oxaliplatin in a stop-and-go fashion in advanced colorectal cancer-a GERCOR study. J Clin Oncol 24: 394-400, 2006.

29. Cremolini C, Loupakis F, Antoniotti C, Lupi C, Sensi E, Lonardi S, Mezi S, Tomasello G, Ronzoni M, Zaniboni A, et al: FOLFOXIRI plus bevacizumab versus FOLFIRI plus bevacizumab as first-line treatment of patients with metastatic colorectal cancer: Updated overall survival and molecular subgroup analyses of the open-label, phase 3 TRIBE study. Lancet Oncol 16: 1306-1315, 2015.

30. Lee KH, Chang HJ, Han SW, Oh DY, Im SA, Bang YJ, Kim SY, Lee KW, Kim JH, Hong YS, et al: Pharmacogenetic analysis of adjuvant FOLFOX for Korean patients with colon cancer. Cancer Chemother Pharmacol 71: 843-851, 2013.

31. Stoehlmacher J, Park DJ, Zhang W, Yang D, Groshen S, Zahedy S and Lenz HJ: A multivariate analysis of genomic polymorphisms: Prediction of clinical outcome to 5-FU/oxaliplatin combination chemotherapy in refractory colorectal cancer. Br J Cancer 91: 344-354, 2004.

32. Kim SH, Kwon HC, Oh SY, Lee DM, Lee S, Lee JH, Roh MS, Kim DC, Park KJ, Choi HJ and Kim HJ: Prognostic value of ERCC1, thymidylate synthase, and glutathione S-transferase pi for 5-FU/oxaliplatin chemotherapy in advanced colorectal cancer. Am J Clin Oncol 32: 38-43, 2009.

33. Ongerboer de Visser BW and Tiessens G: Polyneuropathy induced by cisplatin. Prog Exp Tumor Res 29: 190-196, 1985.

34. Liu YP, Ling Y, Qi QF, Zhang YP, Zhang CS, Zhu CT, Wang MH and Pan YD: Genetic polymorphisms of ERCC1-118, XRCC1-399 and GSTP1-105 are associated with the clinical outcome of gastric cancer patients receiving oxaliplatin-based adjuvant chemotherapy. Mol Med Rep 7: 1904-1911, 2013. 
35. Ott K, Lordick F, Becker K, Ulm K, Siewert J, Höfler H and Keller G: Glutathione-S-transferase P1, T1 and M1 genetic polymorphisms in neoadjuvant-treated locally advanced gastric cancer: GSTM1-present genotype is associated with better prognosis in completely resected patients. Int J Colorectal Dis 23 . 773-782, 2008.

36. Kwon HC, Roh MS, Oh SY, Kim SH, Kim MC, Kim JS and Kim HJ: Prognostic value of expression of ERCC1, thymidylate synthase, and glutathione S-transferase P1 for 5-fluorouracil/oxaliplatin chemotherapy in advanced gastric cancer. Ann Oncol 18: 504-509, 2017.

37. Geng R, Chen Z, Zhao X, Qiu L, Liu X, Liu R, Guo W, He G, $\mathrm{Li}$ J and Zhu X: Oxidative stress-related genetic polymorphisms are associated with the prognosis of metastatic gastric cancer patients treated with epirubicin, oxaliplatin and 5-fluorouracil combination chemotherapy. PLoS One 9: e116027, 2014.
38. Watson MA, Stewart RK, Smith GB, Massey TE and Bell DA: Human glutathione S-transferase P1 polymorphisms: Relationship to lung tissue enzyme activity and population frequency distribution. Carcinogenesis 19: 275-280, 1998.

39. Union for International Cancer Control: TNM Classification of Malignant Tumors. 8th edition. Brierley JD, Gospodarowicz MK and Wittekind C (eds). Wiley-Blackwell, Chichester, 2017. 